\title{
Discussion on New Evaluation Technology of Non-Metallic Composite Continuous Pipe for Oil and Gas Field
}

\author{
Xuehua Cai, Xiaodong Shao, Zhao Zhang, Han Ding, Guoquan Qi, Houbu Li \\ State Key Laboratory of Performance and Structural Safety for Petroleum Tubular Goods and Equipment Materials, CNPC \\ Tubular Goods Research Institute, Xi'an, China \\ Email: caixuehua@cnpc.com.cn
}

How to cite this paper: Cai, X.H., Shao, X.D., Zhang, Z., Ding, H., Qi, G.Q. and Li, H.B. (2021) Discussion on New Evaluation Technology of Non-Metallic Composite Continuous Pipe for Oil and Gas Field. Journal of Materials Science and Chemical Engineering, 9, 1-6.

https://doi.org/10.4236/msce.2021.912001

Received: November 29, 2021

Accepted: December 18, 2021

Published: December 21, 2021

\begin{abstract}
In view of the serious lack and lag of the test and evaluation technology of non-metallic composite continuous pipe, and focusing on the characteristics of the application of non-metallic composite continuous pipe in oil field, this paper discusses a series of new full-scale test and evaluation technologies for accurately evaluating the product quality and practical application performance of non-metallic composite continuous pipe, which effectively solves the major technical problem that the new products of non-metallic pipe cannot be accurately evaluated. Based on the characteristics of the application of non-metallic composite continuous pipe in oil field, a series of new full-scale test evaluation technologies which can accurately evaluate the product quality and practical application performance of non-metallic pipe are designed through a large number of tests. The test and evaluation technology can accurately evaluate the key performance of high and low pressure cycle, high and low temperature cycle, gas permeability resistance, minimum bending radius etc. It provides a scientific evaluation basis for the standardized application of nonmetallic continuous pipe and a reliable quality control method for the selection of products in oil field.
\end{abstract}

\section{Keywords}

Non Metallic Composite Continuous Pipe, Full-Size, High and Low Temperature Cycle, Minimum Bending Radius

\section{Introduction}

With the increase of oil and gas exploration and development in China, the oil field medium environment is becoming more and more complex and harsh, re- 
sulting in more and more serious corrosion problems of steel pipelines [1] [2], which brings great risks to the safe production of the oil field. Non metallic and composite pipes have the advantages of excellent corrosion resistance, good scale and wax resistance, light weight and low later maintenance cost. They have become one of the important solutions to the corrosion problems of oil field pipelines. By the end of 2018, the consumption of non-metallic pipes had exceeded $45,000 \mathrm{~km}$. The large-scale application of non-metallic pipes is of great significance to ensure energy security. However, due to the serious defects such as incomplete and inaccurate evaluation tests in the quality evaluation technology of non-metallic pipe products mainly based on small-scale physical and chemical tests [3] [4], the non-metallic composite continuous pipe products evaluated as "qualified" in the laboratory frequently have problems such as shortening the service life, failure in service for only one year or even a few months [5] [6] [7], It will bring serious consequences to the normal production of the oilfield. Therefore, the industry urgently needs an experimental evaluation technology that can accurately evaluate the product quality of non-metallic composite continuous pipe, especially its full-scale application performance.

\section{Full Scale Evaluation Technology}

\subsection{Evaluation Technology of Circulating Pressure of Non-Metallic Composite Continuous Pipe}

There are often frequent pressure fluctuations in the wellhead output pressure of the oilfield and the gas transmission pipeline. In order to avoid the failure of non-metallic composite continuous pipe such as fatigue or pipe body pulling off from the joint caused by frequent pressure fluctuations, it is necessary to carry out the pressure cycle resistance test of non-metallic composite continuous pipe, determine the pressure cycle technical indicators and put forward prevention and control measures. Use the pressure cycle testing machine to increase or reduce the internal pressure of the non-metallic composite continuous pipe sample with a certain pressure (water pressure) cycle amplitude and frequency, observe whether the sample has structural instability or pressure reduction, and then determine the fatigue resistance of the continuous pipe body and connecting parts to high and low pressure cycles, as shown in Figure 1. The maximum circulating pressure refers to the specific service conditions of on-site gas transmission pipeline and wellhead.

\subsection{Evaluation Technology of Gas Seal Performance of Non-Metallic Composite Continuous Pipe}

When non-metallic composite continuous pipe is transporting natural gas and other gas media, the channeling layer of transported gas molecules will cause pipe foaming failure. Therefore, in order to prevent channeling failure caused by insufficient gas sealing performance in high-pressure and high $\mathrm{H}_{2} \mathrm{~S}$ gas transmission environment, it is necessary to carry out experimental research on the 


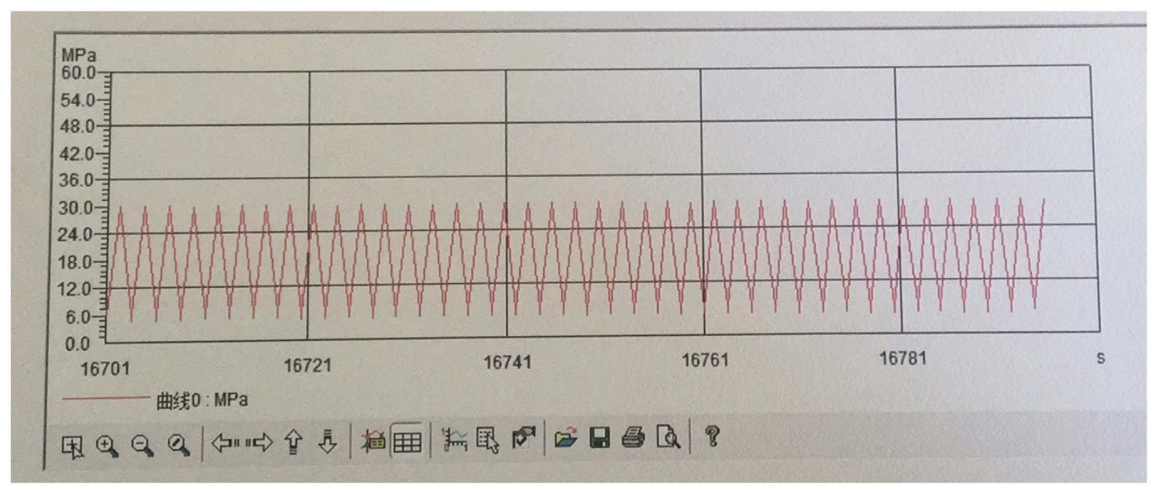

Figure 1. Pressure cycle test curve.

overall gas sealing performance of non-metallic composite continuous pipe, determine the gas sealing technical indicators and put forward prevention and control measures. Testing the gas tightness of non-metallic composite continuous pipe and joint has become the key to avoid the failure of gas transmission non-metallic composite continuous pipe. This project simulates the field application state of the non-metallic composite continuous pipe, places the non-metallic composite continuous pipe sample (including the joint) in the water tank, uses the gas pressurization test system to fill $\mathrm{N} 2$ into the test sample to a certain pressure, maintains the constant pressure state, observes whether the sample has air leakage or pressure reduction, and then determines the gas sealing performance of the composite pipe body and connecting parts, as shown in Figure 2 and Figure 3.

\subsection{High and Low Temperature Cycle Evaluation Technology of Non-Metallic Composite Continuous Pipe}

Based on the high and low temperature characteristics of non-metallic materials, the properties of non-metallic composite continuous pipes will also change significantly under the action of continuous high or low temperature. A large number of studies have been carried out at home and abroad on the influence of low temperature on engineering plastics, rubber and typical non-metallic structural parts. However, the effect of high and low temperature alternating action with a certain frequency and a certain period on non-metallic composite pipes has not been reported. A large number of non-metallic composite continuous pipes have been used in the surface gathering and transportation system of oil fields in Western China. The temperature difference between day and night in the desert environment of oil fields varies greatly. Whether this alternating effect of high and low temperature will affect the size change, pressure bearing performance, tensile performance and external pressure resistance of pipes has attracted widespread attention. Aiming at the problems of large variation of temperature difference between day and night in desert environment of oil field blocks in Western China and unclear impact of high and low temperature alternation and stress disturbance on the performance of non-metallic pipes, this 


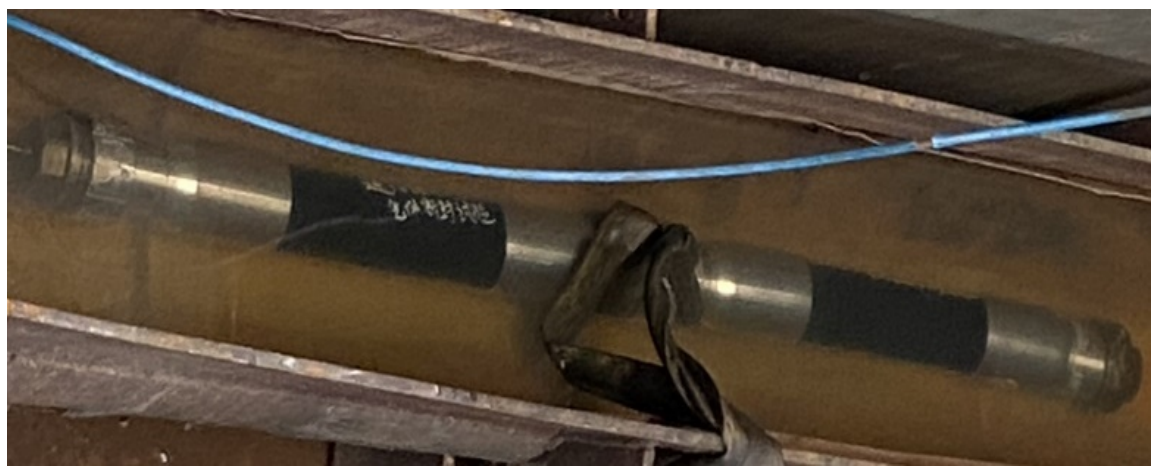

Figure 2. Gas seal test of non-metallic composite continuous pipe.

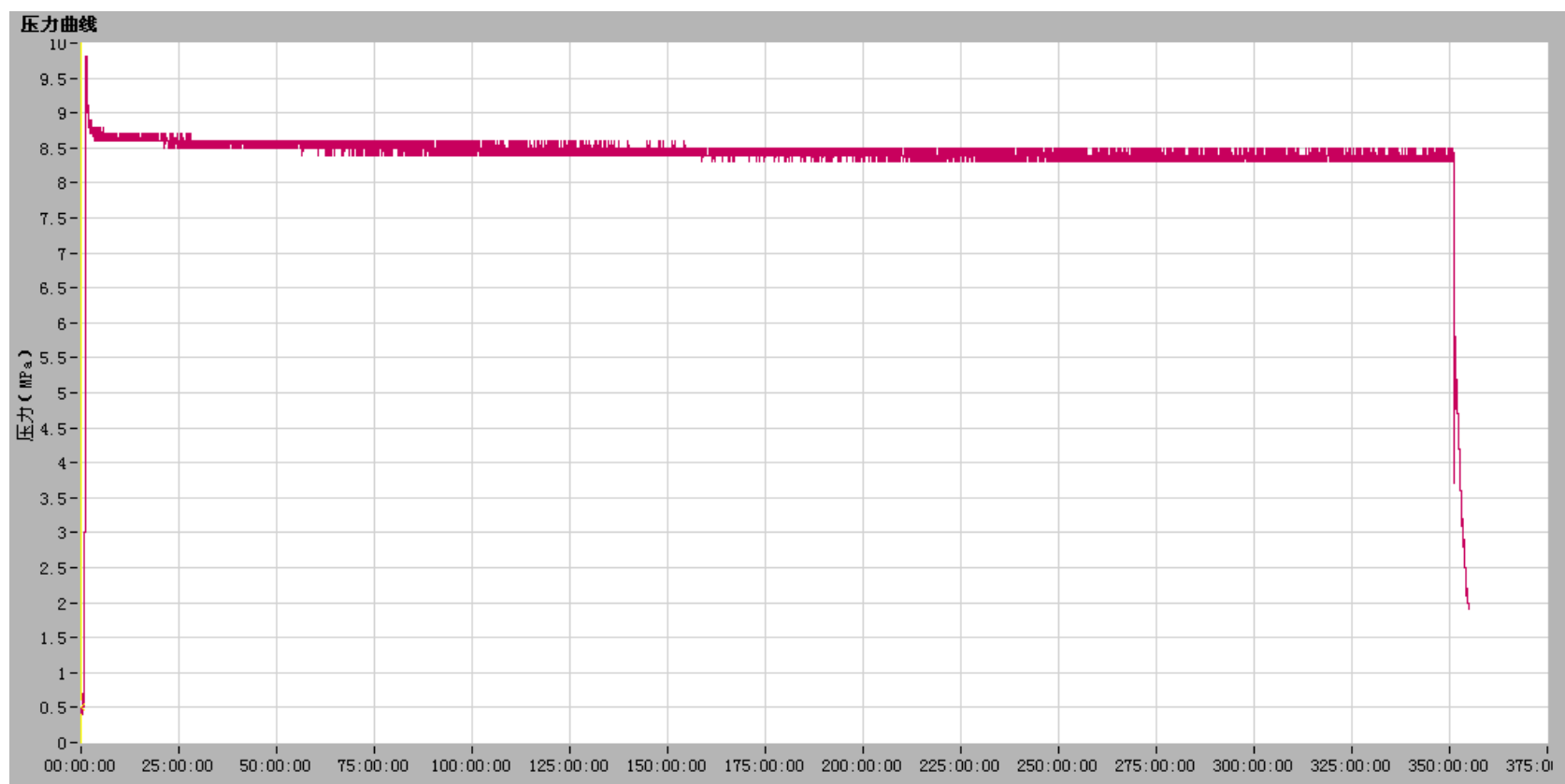

Figure 3. Gas seal test curve of non-metallic composite continuous pipe.

project establishes a high and low temperature alternation test method for nonmetallic pipes, uses this method to simulate the changes of pressure bearing performance and tensile performance of non-metallic pipes under high and low temperature alternation environment, and analyzes the change trend, The influence law of high and low temperature alternating action on the pressure bearing and tensile properties of composite pipe is obtained, as shown in Figure 4. It provides technical support for the safe and long-life selection of non-metallic composite continuous pipeline gathering and transmission pipeline.

\subsection{Evaluation Technology of Minimum Bending Radius of Non-Metallic Composite Continuous Pipe}

In the process of storage, installation and normal use of non-metallic composite continuous pipe, when the bending exceeds its minimum bending radius (including minimum storage bending radius and minimum working bending radius), each component unit of the pipe will be affected by bending, and its per- 
formance index will decline significantly, which will seriously affect the service performance and service life of non-metallic pipe. In order to ensure the correct design of non-metallic composite continuous pipe and ensure that the manufactured pipe can meet the safe service, it is essential to carry out the verification test of full-size minimum bending radius. The project simulates the on-site storage or application state of non-metallic pipes, and determines or verifies the minimum bending radius of non-metallic pipes with the critical value of bursting pressure meeting the requirements of relevant standards. The basic principle of this evaluation method is to bend the test pipe back and forth (according to the minimum storage bending radius), or fix the test pipe on the bending test platform and apply load to bend it to a certain bending radius (according to the minimum working bending radius), Then, the pressure bearing performance of the non-metallic pipe after the bending state is adjusted or under a certain bending radius is tested through the hydrostatic pressure and burst test system, and the minimum storage bending radius and minimum working bending radius of the non-metallic pipe are evaluated by the hydrostatic performance and short-time failure pressure value, as shown in Figure 5.

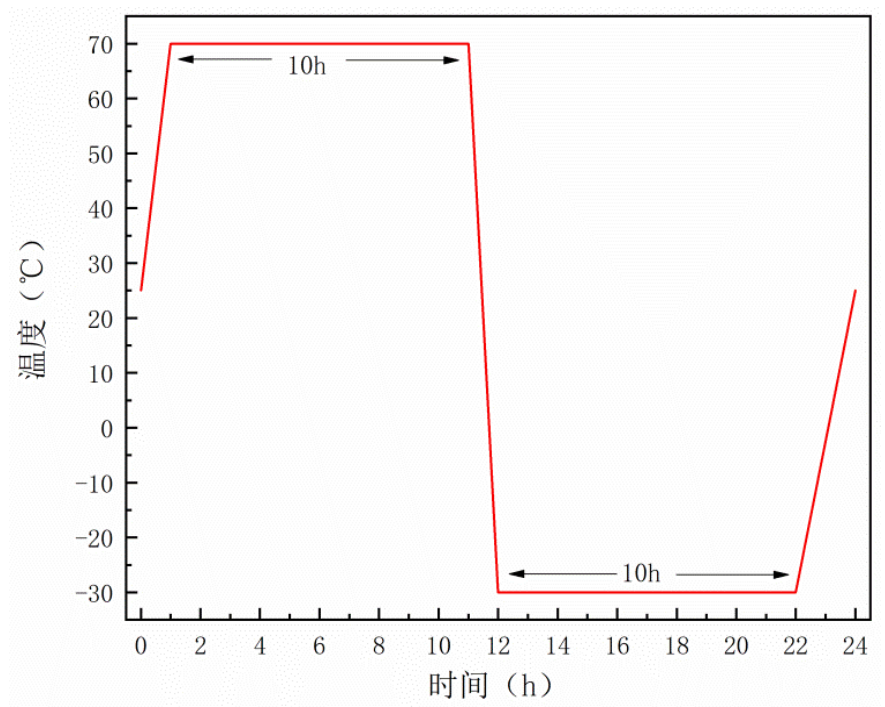

Figure 4. High and low temperature alternating cycle temperature program setting.
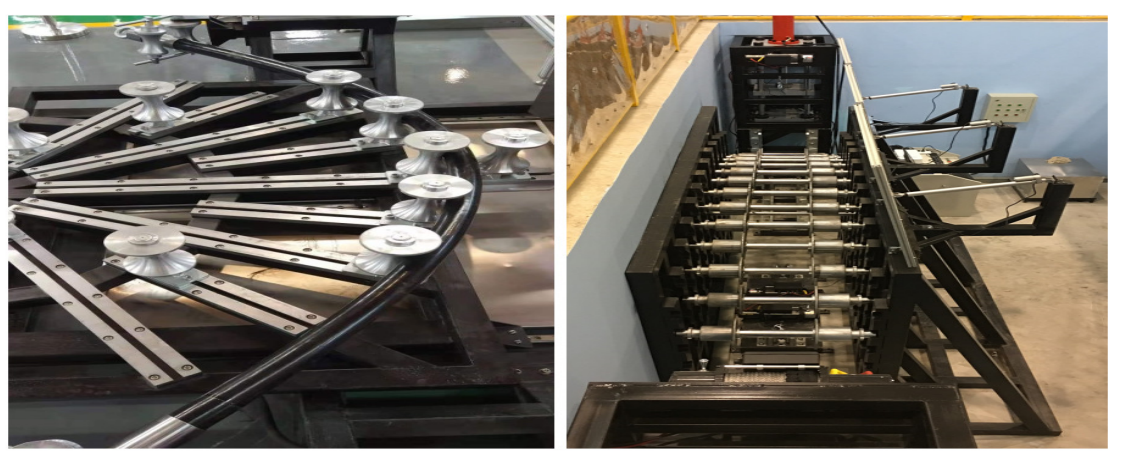

Figure 5. Evaluation of minimum bending radius of full-size non-metallic composite continuous pipe. 


\section{Conclusions and Recommendations}

The developed and designed cyclic pressure evaluation method of non-metallic composite continuous pipe can simulate the pipe fatigue caused by the change of wellhead output pressure. The gas sealing performance evaluation method of non-metallic composite continuous pipe for gas transmission solves the problem of lack of gas sealing performance evaluation method of non-metallic composite continuous pipe in China. The high and low temperature cycle evaluation technology of non-metallic composite continuous pipe is proposed.

It provides a scientific evaluation method for comprehensive evaluation of product quality changes caused by sudden changes in internal transmission medium temperature and large oil field environmental temperature difference. The established evaluation technology of the minimum bending radius of non-metallic composite continuous pipe can provide a scientific inspection means and judgment basis for the performance impact of coil during the production, transportation and laying of non-metallic composite continuous pipe.

The full-scale evaluation method developed and designed by the project has been applied to the quality control process management of non-metallic composite continuous pipe procurement, construction, acceptance and operation in each oilfield, which not only ensures the good applicability of the selected nonmetallic composite continuous pipe, but also helps to control the cost of nonmetallic composite continuous pipe, and effectively reduces the failure probability of subsequent application, It ensures the safe production of oil and gas fields and obtains remarkable economic and social benefits.

\section{Conflicts of Interest}

The authors declare no conflicts of interest regarding the publication of this paper.

\section{References}

[1] Qi, D.T., et al. (2012) Application of Non-Metallic Composite Pipes in Oilfields in China. ICPTT 2012, 283-291. https://doi.org/10.1061/9780784412619.032

[2] Cai, X.H., et al. (2009) Analysis of RTP for Natural Gas Transportation in Changqing Oilfield. ICPTT2009, 2064-2068. https://doi.org/10.1061/41073(361)218

[3] Ding, N. (2010) Hydraulic Tests of Composite Pope Used in Oil and Gasfields. Pipeline Technique and Equipment, 4, 51-54.

[4] Li, H.B. (2016) Present Status and Development Proposals of Standards of Reinforced Thermoplastic Pipes. Plastics, 45, 85-87.

[5] Zhang, X.M. (2020) Simulation of Pressure-Bearing Performance of Polyester Fiber Reinforce Polyethylene Composite Pipes. Engineering Plastics Application, 48, 118 122.

[6] Qi, G.Q. (2015) Evaluation Method of Flexible Composite Pipe under High Temperature and High Pressure Conditions. Petroleum Tubular Goods \& Instruments, 1, 31-33.

[7] Qi, G.Q. (2017) Study on Control of Gas Permeation Behavior in Reinforced Thermoplastics Pipeline. Petroleum Tubular Goods \& Instruments, 3, 58-61. 